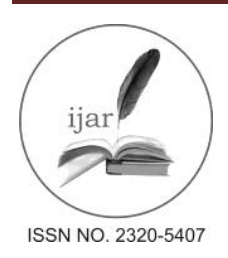

Journal homepage: http://www.journalijar.com
Journal DOI: $10.21474 /$ IJAR01

RESEARCH ARTICLE
INTERNATIONAL JOURNAL

OF ADVANCED RESEARCH

מחיח

\title{
Simultaneous Quantification of T-Cell Receptor Excision Circles (TRECs) and K-Deleting Recombination Excision Circles (KRECs) by Real-time PCR.
}

Lamyaa Salem, Hala A Talkhan, Salwa Bakr, Afaf A. Mostafa, Nesrine A Mohamed. Clinical Pathology and Immunology Faculty of Medicine, Ain Shams University, Egypt.

\section{Manuscript Info}

Manuscript History:

Received: 17 February 2016

Final Accepted: 19 March 2016

Published Online: April 2016

Key words:

Immunodeficiency, T-cell receptor excision circles, K-deleting recombination circles, Newborn screening.

*Corresponding Author

Nesrine A Mohamed

\section{Abstract}

Background: The clinical experience gathered throughout the years endorses primary immunodeficiency diseases (PIDs) awareness and guides research into newborn screening and future therapeutic strategies.Combined T-cell receptor excision circle levels (TRECs) and kappa-deleting recombination excision circles (KRECs) assay paves the way to new potential applications in this field.

Objectives: We aimed to establish a technique for quantification of TRECs and KRECs in Egyptian individuals in our laboratory and to set a lower threshold of normal for TRECs and KRECs in pediatric population for different age groups as a start for its implementation in newborn screening protocols for PIDs.

Methods: 50 apparently healthy children ( 25 males and 25 females) with age ranging from 1 day to 16 years were analyzed..Combined quantification of TRECs and KRECs in the genomic DNA of peripheral blood mononuclear cells was performed using real-time quantitative PCR.

Results: Individuals in the study were divided in to 5 different age groups Data regarding lower threshold of normal for TRECs and KRECs copies per $\mathrm{ml}$ of blood among the study group was obtained. A highly significant negative correlation between TRECs and KRECs, both calculated per $10^{6}$ PBMCs and per $\mathrm{ml}$ of blood and age was observed. On the contrary, there was no statistically significant differences in the studied parameters between males and females when evaluated regardless of age ( $p$ value $=0.697$ ).

Conclusion: It appeared that it is technically feasible to introduce the TRECs/KRECs quantitation by real time PCR into routine laboratory practice to be used in the near future both for new born screening for PIDs

Copy Right, IJAR, 2016,. All rights reserved.

\section{Introduction:-}

Primary immunodeficiency diseases (PIDs) are a group of disorders with defects in the development or function (or both) of the immune system. The global prevalence of PIDs varies between 0.3 and 12 per 100000 population, and is higher in areas with high rates of consanguinity [1].

In Egypt, no national registry of PIDs exists and only 160 Egyptian PID children were registered with the European Society for Immunodeficiencies (ESID). These patients were affiliated with two PIDs centers in the country: one at Ain Shams University (100 cases) and the other at Cairo University (60 cases) patients. So clearly, the actual burden of PIDs in Egypt is underestimated [2].

Children with PIDs tend to have recurrent bacterial or fungal infections[3].Severe combined immunodeficiency (SCID) in particular, is fatal in infancy unless affected infants can be diagnosed before the onset of devastating infections and provided with an immune system through allogenic hematopoietic stem cell transplantation (HSCT), enzyme replacement, or gene therapy[4]. 
T- and B-lymphocytes are unique in their ability to create a receptor by genomic rearrangement of their antigen receptor genes via $\mathrm{V}(\mathrm{D}) \mathrm{J}$ recombination. On one hand, DNA strand breakage during the thymic and bone marrow (BM) maturation processes of the $\mathrm{T}$ cell receptor (TCR) and the $\mathrm{B}$ cell receptor (BCR), creates functional receptors (i.e., the formation of coding joint recombination sites), while, on the other hand, it creates by products termed TCR excision circles (TRECs) and kappa-deleting recombination excision circles (KRECs), respectively [5].

TRECs and KRECs are small circularized DNA elements. They have no function, but because they are stable and cannot be replicated, they are diluted after each cell division, thus persisting only in one of the two daughter cells. Therefore, their levels in the peripheral blood can be assumed as an estimate of the thymic and BM output [6].

The measure of TRECs and KRECs which can be reliably performed using real-time quantitative PCR has contributed to an improved characterization of PIDs, to the identification of patients' subgroups, and to the monitoring of stem cell transplantation and enzyme replacement therapy. For the same diseases, the TREC and KREC assays, introduced in the newborn screening program, allow early disease identification and may lead to discovery of new genetic defects [7].

\section{Materials and methods:- \\ Subjects:-}

This study was conducted at Ain Shams University Hospitals. Fifty apparently healthy children and adolescents between the ages of 1 and 16 years were selected for the study. They were recruited during routine pediatrician outpatient visits for conditions not related to immunologic or infectious diseases or during preoperative evaluation for minor surgical procedures, such as circumcision and inguinal hernia. All healthy control patients underwent thorough clinical evaluations and were screened for a normal blood cell count. Exclusion criteria included the presence of signs or symptoms of primary immunodeficiency disease based on the " 10 Warning Signs of Primary Immunodeficiency" (Jeffrey Modell Foundation) and the presence of active bacterial or viral infections. Patients with allergic conditions and those with premature birth were also excluded. This study was approved by the research ethics committee of Ain Shams University (FMASU 1524/2013). A written consent was obtained from the parents of all children included in the study. The laboratory work was performed at the clinical pathology department of Ain Shams University Hospitals and Centro di Ricerca AIL- Spedali Civili Di Brescia, Italy

\section{Methods:-}

Five $\mathrm{mL}$ of venous blood was drawn using EDTA Vacutainer tubes. Peripheral blood mononuclear cells (PBMCs) were isolated by Ficoll-Hypaque (Lymphoprep, Axis-Shield, Scotland) density gradient centrifugation. Then, PBMCs were frozen at $-20^{\circ} \mathrm{C}$ for subsequent DNA extraction.

DNA was extracted using the QIAamp DNA Blood Mini Kit (Qiagen, Valencia, CA), according to the manufacturer's instructions. The number of TRECs and KRECs was simultaneously detected by a duplex Real-Time PCR assay performed using the Rotor Gene Q MDx (QIAGEN, Hilden, Germany). SJ TRECs forward primer (5'CAC ATC CCT TTC AAC CAT GCT-3') and probe (5'-FAM-ACA CCT CTG GTT TTT GTA AAG GTG CCC ACT- TAMRA-3') were those designed by Douek et al. [14], while the reverse primer for SJ TRECs (5'-TGC AGG TGC CTA TGC ATC A- 3'), together with the forward (5'-TCC CTT AGT GGC ATT ATT TGT ATC ACT-3') and reverse (5'-AGG AGC CAG CTC TTA CCC TAG AGT-3') primers and probe (5'-HEX-TCT GCA CGG GCA GCA GGT TGG-TAMRA-3') for SJ KRECs and the oligonucleotides and probe for TRAC gene (forward 5'-TGG CCT AAC CCT GAT CCT CTT-3', reverse 5'-GGA TTT AGA GTC TCT CAG CTG GTA CAC-3' and probe 5'FAM-TCC CAC AGA TAT CCA GAA CCC TGA CCC- TAMRA-3') were designed by Sottini et al.[8].

PCR reactions were developed in a final volume of a $25-\mu 1$ mixture, consisting of $5 \mu 1$ (400-500 ng) of genomic DNA solution, $12.5 \mu \mathrm{l}$ of $2 \times$ TaqMan Universal PCR master mix consisting of $1 \mathrm{x}$ Buffer, $1.5 \mathrm{mM} \mathrm{MgCl}$, dNTP mix (200 $\mu \mathrm{M}$ each), Amplitaq DNA polymerase $(2.5 \mathrm{U} / 25 \mu \mathrm{l})$, and primers and probes for SJ TRECs and SJ KRECs

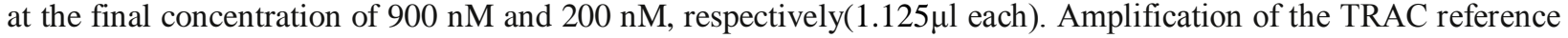
gene was done in the same plate with the same concentrations of TRAC- specific primers and probe. The assay was run for the three genes with the same standard protocol consisting of an initial heating at $95^{\circ} \mathrm{C}$ for $10 \mathrm{~min}$, followed by 45 cycles of denaturation at $95{ }^{\circ} \mathrm{C}$ for $15 \mathrm{~s}$ and a combined primer/probe annealing and elongation at $60{ }^{\circ} \mathrm{C}$ for 1 min. TRECs, KRECs and TRAC number has been obtained by extrapolating the respective sample quantities from 
the standard curve obtained by serial dilutions $\left(10^{6}, 10^{5}, 10^{4}, 10^{3}, 10^{2}\right.$, and 10) of the linearized triple-insert plasmid DNA which were amplified in each PCR plate. A standard curve was included in every PCR reaction.

The triple-insert plasmid DNA was kindly supplied by Dr Luisa Imberti and Dr Alessandra SottiniLaboratorioCrea, SpedaliCivili Di Brescia, Italy.

The number of TRECs or KRECs per $10^{6}$ PBMC was calculated using the following formula:

TRECs or KRECs quantity

TRAC quantity $/ 2$

The quantity of TRAC had to be divided for 2 because in each cell there are two TRAC gene copies, e.g. one for each chromosome. This value, together with the lymphocyte plus monocyte count (which are the cells obtained in PBMCs preparation) in $1 \mathrm{ml}$ of blood, was utilized to calculate the number of TRECs or KRECs per ml of blood $($ copies $/ \mathrm{ml})$ that is $=\left(\right.$ TRECs or KRECs per $\left.1 \times 10^{6} \mathrm{PBMC}\right) \times($ lymphocyte plus monocyte count in $1 \mathrm{ml}$ of blood $) / 10^{6}$.

\section{Statistical analysis:-}

Analysis of the data was performed using the SPSS program, version 23. Data were expressed as median and interquartile range (IQR) for quantitative non-parametric measures and both number and percentage for categorized data .Mann-Whitney U test was used for comparison between two independent groups for non-parametric data. Comparison between more than 2 patient groups for non-parametric data was done using Kruskall Wallis test. Chisquare test was used to compare categorical data. To assess the strength of relationship between the studied parameters and age, Spearman's correlation coefficient test was performed. A $p$ value of less than 0.05 was considered significant.

\section{Results:-}

This study included 50 apparently healthy individuals ( 25 males and 25 females) with age ranging from 1 day to 16 years (median 2.4 years). Individuals included in the study were divided according to the age into appropriate quartiles(Q); Q1 from minimum to $25^{\text {th }}$ Percentile; Q2 from $25^{\text {th }}$ Percentile till $50^{\text {th }}$ Percentile; Q3 from $50^{\text {th }}$ Percentile to $75^{\text {th }}$ Percentile and the last Q4 from $75^{\text {th }}$ Percentile to Maximum (Table 1).

There was a highly significant negative correlation between TRECs and KRECs, both calculated per $10^{6}$ PBMCS and per $\mathrm{ml}$, and age.

There was no statistical significant difference between the four quartiles regarding sex distribution (Table 2). Additionally, there was no significant difference between males and females as regards TRECs and KRECs $(p$ value $=0.697$ ).

In an attempt to establish a reference range for TRECs and KRECs, subjects were divided in to 5 different age groups and the following results were obtained as lower threshold of normal range for the studied parameters TRECs copies per $\mathrm{ml}$ of blood: 25172 for the group aged ( 1 day to 5 months); 23989 for the group aged (5 months to 2.4 years); 8294 for the group aged (2.4 years to 5.2 years); 8122 for the group aged (6 years to 9.5 years) and 4670 for the group aged(9.5 to 16 years)(figure 1)

However, results as lower threshold of normal range for KRECs copies per $\mathrm{ml}$ of blood were 30885 for the group aged (1 day to 5 months); 52230 for the group aged (5 months to 2.4 years); 32139 for the group aged (2.5 years to 4 years); 6633 for the group aged (4 years to 5 years) and 2198 for the group aged (5.2 years to 16 years)(figure 2). 


\section{Discussion:-}

The set up of a duplex quantitative real time PCR by measuring both TRECs and KRECs, eliminates the variability associated with DNA quantification by real-time PCR by the use of a unique standard curve obtained by diluting a triple-insert plasmid containing fragments of TRECs, KRECs and T-cell receptor alpha constant (TCRAC) gene in a 1:1:1 ratio. This allows a more accurate evaluation of the TREC and KREC copy number and reagent cost reduction [8].

The assay can be useful to measure the extent of T- and B-cell neo-production in patients with SCID, CVID, autoimmune diseases, HIV infection and to monitor the immune recovery after HSCT [9].

The combined TRECs and KRECs assay can be also used to detect immunodeficiencies in newborn screening programs. In this case, the test must be performed on DNA extracted from small spots of blood blotted and dried on filter paper (Guthrie cards), must be highly sensitive and specific for the target diseases, as well as highly reproducible and cost-effective[10].

Over the years measurement units of TREC concentration varied including TRECs per CD45RA+ T cell, TRECs/ $\mu \mathrm{g}$ DNA of T cells, TRECs $/ 10^{5}$ CD4+ T cells, TRECs $/ 10^{6}$ PBMCs and TRECs $/ \mathrm{ml}$ of blood [11].

The interpretation of TRECs calculated per $10^{6} \mathrm{PBMCs}$ may be complicated by the dilution effects of peripheral Tcell divisions. This problem can be overcome by expressing TRECs per $\mathrm{ml}$ of blood [12].

The number of TRECs or KRECs per $10^{6} \mathrm{PBMCs}$ was calculated by dividing the quantity of TRECs or KRECs by the number of TCRAC gene copies, which has to be divided by 2 because there are two TCRAC gene copies in each cell (i.e. one for each chromosome) and then multiplied by $10^{6}$. This value, together with the lymphocyte plus monocyte count (which are the cells obtained in the PBMCs preparation) in $1 \mathrm{~mL}$ of blood, was used to calculate the number of TRECs or KRECs per $\mathrm{mL}$ of blood [8].

In the final calculations, a non-perfect equivalence between lymphocyte plus monocyte count as an estimate of the PBMC quantity may add some bias in the conversion between values expressed per $10^{6} \mathrm{PBMCs}$ and values expressed per $\mathrm{ml}$ of blood [7].

The result interpretation can be affected by some issues. First, the reference ranges could change with gender and age. Furthermore, in case of certain immune deficiencies or autoimmune syndromes, a homeostatic peripheral replication of existing memory cells can be present, which dilutes TREC/KREC content. This may lead to the erroneous conclusion that the thymic/bone marrow output is lower than it actually is. Therefore, it is advisable to always estimate lymphocyte neoproduction by also calculating TRECs/KRECs per $\mathrm{ml}$ of blood, which makes results independent of the proliferation rate of memory cells. Third, whatever is the unit of measure, the test result may lead to biased overestimates, due to persistence of some long-lived naïve lymphocytes [13].

In this study, a decline in TRECs copies/ $\mathrm{ml}$ of blood with increasing age in accordance with Douek et al. [14] and Zhang et al. [15] was demonstrated. Although, Douek et al. [14] demonstrated a sharp decline in TREC levels with age, Zhang et al. [15] demonstrated a gradual decline in TREC levels, with stable thymic output within the first 5 years of life.

Similarly, Steffens et al.[16] reported an inverse relation between age and TREC production in healthy donors, especially in the age ranges 0 to 5 years. In addition, Chen et al.[17] found that there was a statistically significant inverse correlation between TREC numbers and age in a study on 77 healthy sibling donors aged (1-20 years).

Additionally, Sottini et al.[8] proposed that the number of TRECs decreases with age due to thymic involution and the number of KRECs decreases with the age with a pattern similar to TRECs only in children, whereas in the adults the bone marrow output is fairly stable throughout life.

Interestingly in this study, a variability in the number of TREC copies in children and adolescents of the same age was found. However, this variability has been previously observed by Steffens et al.[16], Wu et al.[19], Chen et al.[17]and Arismendi et al.[18] and has been attributed to genetic factors and inherent differences in the proportion of thymic tissue in healthy patients of the same age. 
In the present study, no statistically significant differences in the parameters KRECs and TRECs copies per million of cells and KRECs and TRECs copies per ml of blood between males and females when evaluated regardless of age was demonstrated $(\mathrm{p}=0.697)$. Our results were in agreement with Chen et al.[17]., Arismendi et al.[18]and Sottini et al.[8]

In an attempt to establish a reference range for TRECs and KRECs in healthy individuals with age ranging from 1 day-16 years, the 50 subjects included in this study were divided in to 5 different age groups and the following results were obtained as lower threshold of normal range for the studied parameters TRECs copies per ml of blood: 25172 for the group aged (1 day to 5 months); 23989 for the group aged ( 5 months to 2.4 years); 8294 for the group aged (2.4 years to 5.2 years); 8122 for the group aged (6 years to 9.5 years) and 4670 for the group aged(9.5 to 16 years).

However, results as lower threshold of normal range for KRECs copies per ml of blood were 30885 for the group aged ( 1 day to 5 months); 52230 for the group aged ( 5 months to 2.4 years); 32139 for the group aged (2.5 years to 4 years); 6633 for the group aged (4 years to 5 years) and 2198 for the group aged (5.2 years to 16 years).

Similarly, Chen et al.[17] established a normal range of TRECs production in PBMCs by examining 77 healthy sibling donors (33 female, 44 male) within the same age range as the patients in his study (1-20 years). The TRECs count ranged from 1200 to 155000 copies/mL blood among healthy donors. The mean TREC number was 24015 \pm 2737 copies $/ \mathrm{mL}$ blood. The lowest value obtained for TREC from healthy donors (1200 copies/mL blood) was established as the lower threshold of the normal range. However they calculated TREC copies/ml of blood as mean \pm SE while in this study we calculated median and percentiles.

In the study by Arismendi et al.[18] in which95 healthy children and adolescents were analyzed(age range: $1-18$ years, mean age 9 years). The mean TREC count in PBMCs in all individuals was $8.9 \times 10^{4} \pm 3.6 \times 10^{4} \mathrm{TRECs} / \mu \mathrm{g}$ DNA. In this study they calculated TRECs/ $\mu \mathrm{g}$ DNA while in this study we calculated TRECs copies/ ml of blood.

In the work done by Sottini et al.[8] combined TRECs and KRECs quantification by real time PCR using triple insert plasmid dilutions as standard curve was performed in a representative sample of 42 children aged 0 - 16 years (male/females: 25/17). Results were obtained as TRECs and KRECs per PBMC, and then the TRECs and KRECs per $\mathrm{ml}$ of blood were calculated.

The cut-off values for TRECs and KRECs were established in male and female controls aged from 0-3 years (TRECs: 32028 copies / $\mathrm{ml}$ and KRECs: 93810 copies/ ml) and those aged from 3-16 years (TRECs: 7770 copies / $\mathrm{ml}$ and KRECs: 1655 copies / $\mathrm{ml})$.

The work done in this study demonstrated that the number of TRECs and KRECs copies decline with age. Additionally, quantification of the numbers of TRECs and KRECs, obtained by the assay of combined TRECs and KRECs quantification by real time PCR can be easily introduced into routine laboratory practice and is highly informative. 


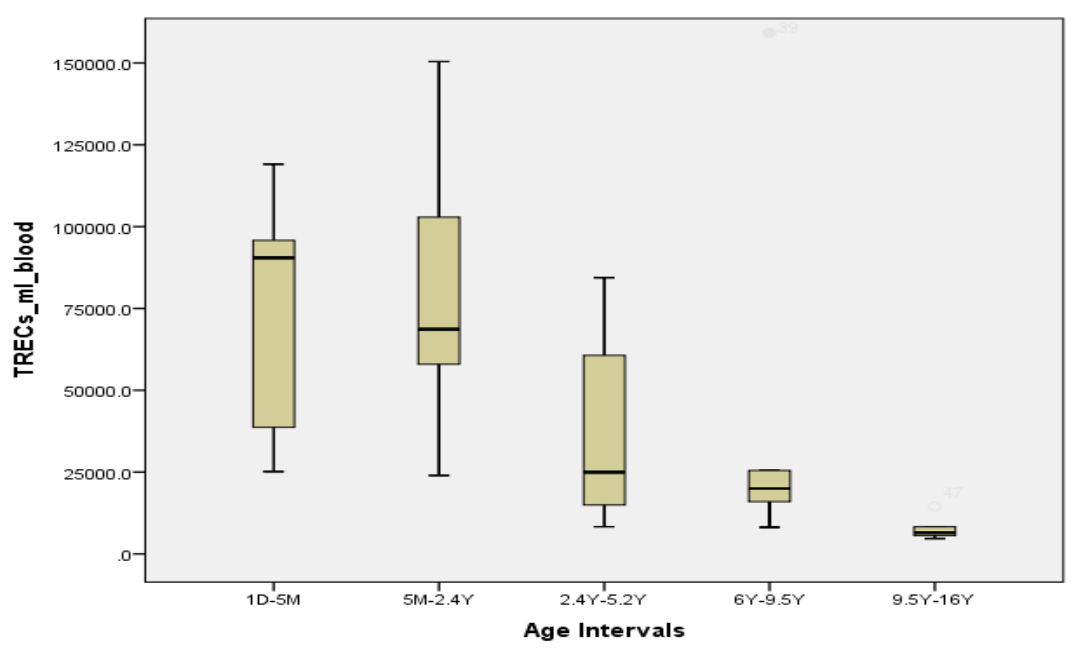

Figure.(1): Box-Plot Chart showing TRECs copies/ml of blood among the Study Age Groups

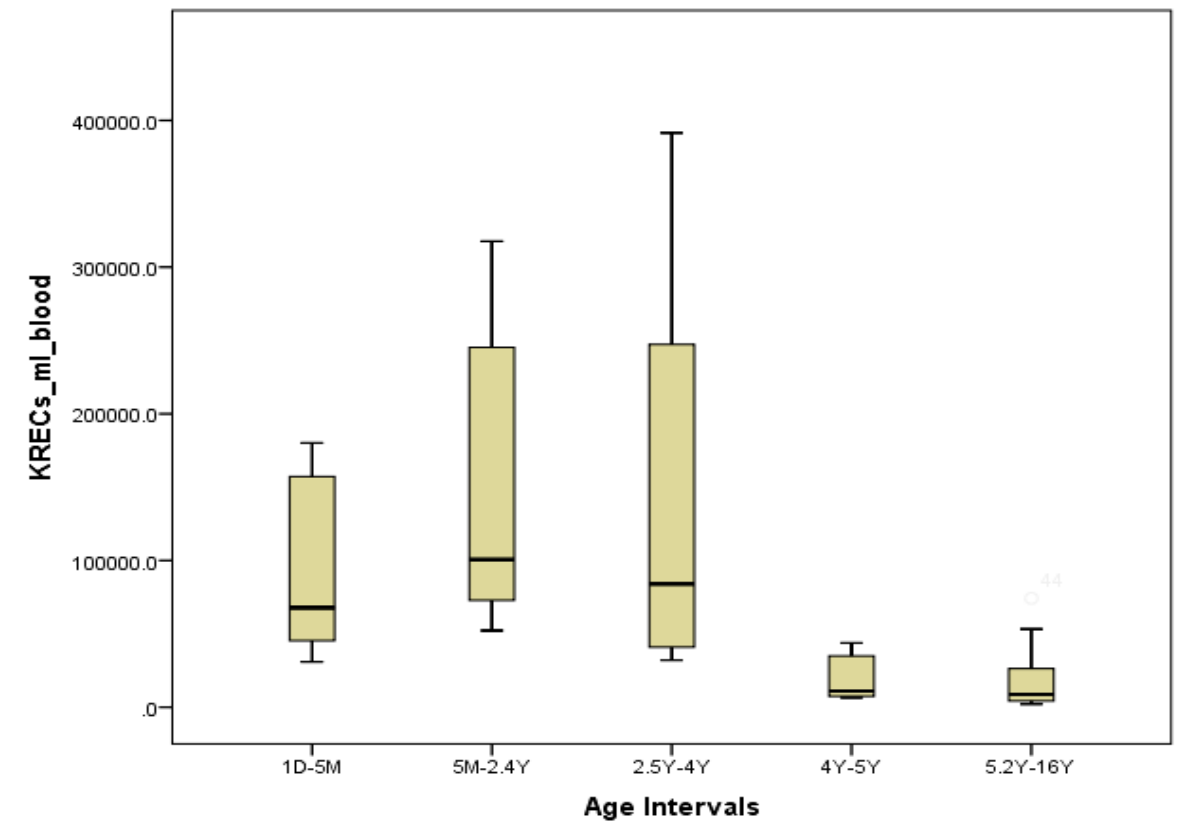

Figure. (2): Box-Plot Chart showing KRECs copies/ ml of blood among Study Age Groups

Table 1: Descriptive statistics of subjects in the study according to age

\begin{tabular}{|l|c|c|c|}
\hline & & \multicolumn{2}{|c|}{ Age } \\
\hline Q1: & n & Minimum & Maximum \\
\hline Q2: & 13 & $1 \mathrm{D}$ & $2.4 \mathrm{Y}$ \\
\hline Q3: & 12 & $5 \mathrm{M}$ & $5.2 \mathrm{Y}$ \\
\hline Q4: & 13 & $2.4 \mathrm{Y}$ & $16 \mathrm{Y}$ \\
\hline
\end{tabular}

Q: Quartile, n: number of subjects, D: day, M: month, Y: year 
Table (2): Statistical comparison between the four studied quartiles regarding sex distribution.

\begin{tabular}{|c|c|c|c|c|c|c|}
\hline $\begin{array}{ll} & \text { Quartiles } \\
\text { Sex } & \\
\end{array}$ & $\begin{array}{c}\text { Q1 } \\
\mathrm{N}(\%) \\
\end{array}$ & $\begin{array}{c}\text { Q2 } \\
\mathrm{N}(\%) \\
\end{array}$ & $\begin{array}{c}\text { Q3 } \\
\mathrm{N}(\%) \\
\end{array}$ & $\begin{array}{c}\text { Q4 } \\
\mathrm{N}(\%)\end{array}$ & $x^{2}$ & $\mathbf{p}$ \\
\hline $\begin{array}{l}\text { Females } \\
\mathrm{N}=25(50 \%)\end{array}$ & $5(38.5)$ & $7(58.3)$ & $6(46.2)$ & $7(58.3)$ & \multirow[t]{3}{*}{$1.436^{\mathrm{a}}$} & \multirow[t]{3}{*}{$0.697(\mathrm{NS})$} \\
\hline $\begin{array}{l}\text { Males } \\
\mathrm{N}=\mathbf{2 5}(\mathbf{5 0 \%})\end{array}$ & $8(61.5)$ & $5(41.7)$ & $7(53.8)$ & $5(41.7)$ & & \\
\hline $\begin{array}{l}\text { Total } \\
\mathrm{N}=\mathbf{5 0}(\mathbf{1 0 0 \%})\end{array}$ & $13(100)$ & $12(100)$ & $13(100)$ & $12(100)$ & & \\
\hline
\end{tabular}

${ }^{\mathrm{a}}$ Chi-square test, NS non-significant

\section{Conclusion:-}

TREC and KREC quantification can be considered a good estimate of recent thymic and bone marrow output as it ensures highly accurate quantitative results because unknown sample quantities are interpolated from standard curves built upon known amounts of starting material. Moreover, it appeared that it is technically feasible to introduce the TRECs/KRECs assay into routine laboratory practice and it can be used in the near future both for new born screening and for a more critical monitoring of the rate of $\mathrm{T}$ - and $\mathrm{B}$-cell immune reconstitution following HSCT.

\section{Ethical Approval:-}

Written informed consent was obtained from each of the participants after approving the study protocol by the Ethics Committee of Faculty of Medicine, Ain Shams University.

\section{Conflict of Interests:-}

There is no conflict of interests.

\section{Acknowledgment:-}

The authors are indebted to DrAlessandra Sottini and Dr Luisa ImbertiCentro di Ricerca AIL- SpedaliCivili Di Brescia, Italy for kindly supplying a TREC-KREC-TRAC plasmid and also for providing TREC/KREC assay training. We thank you for your excellent technical guidance and support.

This work was supported by Science and Technology Development Fund STDF-STF (6114).

\section{References:-}

1. Buldeo S. (2012): Primary immune deficiency diseases. Journal of continuing medical education; 30 (8): $278-$ 281.

2. Reda SM., El-Ghoneimy DH. and Afifi HM. (2013): Clinical Predictors of Primary Immunodeficiency Diseases in Children. Allergy Asthma Immunol Res.;5(2):88-95.

3. Reust CE. (2013): Evaluation of primary immunodeficiency disease in children. Am Fam Physician;87(11):773-778.

4. Puck JM. (2011): The case for newborn screening for severe combined immunodeficiency and related disorders. Ann N Y Acad Sci.;1246:108-117.

5. Ye P. and Kirschner DE. (2002): Reevaluation of T cell excision circles as a measure of human recent thymic emigrants.J Immunol; 168:4968-4979.

6. Sottini A., Serana F., Bertoli D., Chiarini M., Valotti M., Tessitore MV. and Imberti L. (2014): Simultaneous Quantification of T-Cell Receptor Excision Circles (TRECs) and K-Deleting Recombination Excision Circles (KRECs) by Real-time PCR, J. Vis. Exp. (94): e52184.

7. Serana F., Chiarini M., Zanotti C., Sottini A., Bertoli D., Bosio A., Caimi L. and Imberti L. (2013): Use of $\mathrm{V}(\mathrm{D}) \mathrm{J}$ recombination excision circles to identify $\mathrm{T}$ - and B-cell defects and to monitor the treatment in primary and acquired immunodeficiencies. Journal of Translational Medicine, 11:119.

8. Sottini A., Ghidini C., Zanotti C., Chiarini M., Caimi L., Lanfranchi A., Moratto D., Porta F. and Imberti L. (2010): Simultaneous quantification of recent thymic T-cell and bone marrow B-cell emigrants in patients with primary immunodeficiency undergone to stem cell transplantation. Clinical Immunology; 136(2):217-227. 
9. Zanotti C1., Chiarini M., Serana F., Sottini A., Garrafa E., Torri F., Caimi L., Rasia S., Capra R. and Imberti L. (2012): Peripheral accumulation of newly produced $\mathrm{T}$ and $\mathrm{B}$ lymphocytes in natalizumab-treated multiple sclerosis patients. Clin. Immunol;145 (1): 19-26.

10. Borte S., Janzi M., Pan-Hammarstro“m Q ., von Do“beln U ., Nordvall L., Winiarski j., Fasth A. and Hammarstro im L. (2012): Placental Transfer of Maternally-Derived IgA Precludes the Use of Guthrie Card Eluates as a Screening Tool for Primary Immunodeficiency Diseases. PLoS One; 7(8): e43419.

11. Geenen V., Poulin JF., Dion ML., Martens H. et al. (2003): Quantification of T cell receptor rearrangement excision circles to estimate thymic function: an important new tool for endocrine-immune physiology. $J$ Endocrinol; 176:305-311.

12. Ribeiro RM. and Perelson AS. (2007): Determining thymic output quantitatively: using models to interpret experimental T-cell receptor excision circle (TREC) data. Immunol. Rev. 216: 21-34.

13. De Boer RJ. and Perelson AS. (2013): Quantifying T lymphocyte turnover. Journal of Theoretical Biology; 327: 45-87.

14. Douek DC., McFarland RD., Keiser PH., Gage EA., Massey JM., Haynes BF., Polis MA., Haase AT., Feinberg MB., Sullivan JL., Jamieson BD., Zack JA., Picker LJ. and Koup RA. (1998): Changes in thymic function with age and during the treatment of HIV infection. Nature; 396:690-695.

15. Zhang L., Lewin SR., Markowitz M., Lin HH., Skulsky E., Karanicolas R., He Y., Jin X., Tuttleton S., Vesanen M., Spiegel H., Kost R., van Lunzen J., Stellbrink HJ., Wolinsky S., Borkowsky W., Palumbo P., Kostrikis LG. and Ho DD. (1999): Measuring Recent Thymic Emigrants in Blood of Normal and HIV-1infected Individuals before and after Effective Therapy J. Exp. Med:190(5):725-732.

16. Steffens CM., Al-Harthi L., Shott S., Yogev R. and Landay A. (2000): Evaluation of Thymopoiesis Using T Cell Receptor Excision Circles (TRECs): Differential Correlation between Adult and Pediatric TRECs and Na1 ve Phenotypes, Clinical Immunology;97(2): 95-101.

17. Chen, X., Barfield, R., Benaim, E., Leung, W., Knowles, J., Lawrence D. et al. (2005): Prediction of T-cell reconstitution by assessment of $\mathrm{T}$-cell receptor excision circle before allogeneic hematopoietic stem cell transplantation in pediatric patients. Blood; 105: 886-893.

18. Arismendi MI., Kalla's EG., Almeida B., dos Santos BAN., Carneiro-Sampaio MMS and Kayser C. (2012): Thymopoiesis and regulatory $T$ cells in healthy children and adolescents.Clinics;67(5):425-429.

19. Wu CJ., Chillemi A., Alyea EP., Orsini E., Neuberg D., Soiffer RJ. and Ritz J. (2000): Reconstitution of Tcell receptor repertoire diversity following $\mathrm{T}$ cell depleted allogeneic bone marrow transplantation is related to hematopoietic chimerism. Blood; 95:352-359. 